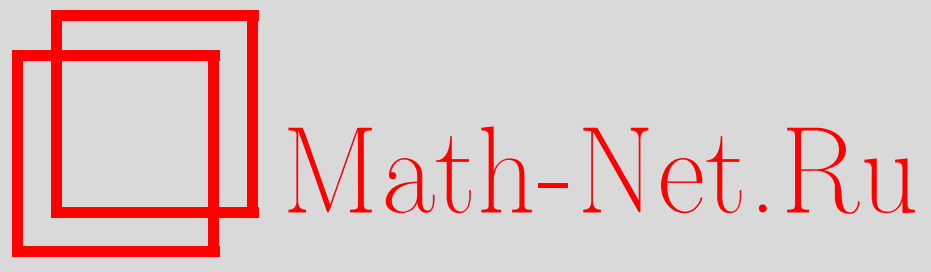

М. Е. Жуковский, Спектры формул первого порядка малой кванторной глубины, УМН, 2015, том 70, выпуск 6, 209-210

DOI: https://doi.org/10.4213/rm9664

Использование Общероссийского математического портала Math-Net.Ru подразумевает, что вы прочитали и согласны с пользовательским соглашением http://www . mathnet.ru/rus/agreement

Параметры загрузки:

IP: 3.89 .185 .249

26 апреля 2023 г., 12:07:07

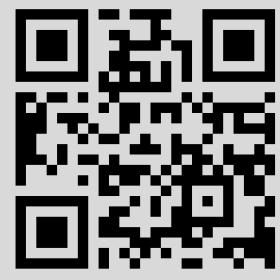




\section{Спектры формул первого порядка малой кванторной глубины}

\section{М. Е. Жуковский}

Асимптотическое поведение вероятностей свойств первого порядка случайного графа Эрдёша-Реньи $G\left(n, n^{-\alpha}\right), \alpha>0$, в контексте законов нуля или единицы было широко изучено в работах [1]-[7]. Прежде чем перейти к формулировкам соответствующих результатов, напомним основные определения.

Пусть $n \in \mathbb{N}, 0 \leqslant p \leqslant 1$. Рассмотрим множества $\Omega_{n}=\left\{G=\left(V_{n}, E\right)\right\}$ всех неориентированных графов без петель и кратных ребер на множестве вершин $V_{n}=$ $\{1,2, \ldots, n\}$. Случайный граф Эрдёша-Ренъи [8], [9] - это случайный элемент $G(n, p)$, заданный на некотором вероятностном пространстве с вероятностной мерой $\mathrm{P}$ и принимающий значения в множестве $\Omega_{n}$, с распределением $\mathrm{P}_{n, p}$ на $\mathcal{F}_{n}=2^{\Omega_{n}}$, определенным следующим образом: $\mathrm{P}_{n, p}(G)=p^{|E|}(1-p)^{C_{n}^{2}-|E|}$. Будем обозначать $\{G(n, p) \models L\}$ событие "граф $G(n, p)$ обладает свойством $L$ ".

Говорят, что случайный граф подчиняется закону нуля или единицы, если вероятность выполнения каждого свойства первого порядка (см. [9], [10]) стремится либо к 0, либо к 1. Случайный граф подчиняется $k$-закону нуля или единицы, если вероятность выполнения каждого свойства, записываемого с помощью формулы первого порядка с кванторной глубиной, не превосходящей $k$ (см. [10]), стремится либо к 0, либо к 1 . Обозначим $\mathcal{L}$ и $\mathcal{L}_{k}$ множество всех свойств первого порядка и множество всех свойств, записываемых с помощью формул первого порядка с кванторной глубиной, не превосходящей $k$, соответственно.

В [1] показано, что если $p=n^{-\alpha+o(1)}, \alpha \in \mathbb{R}_{+} \backslash \mathbb{Q}$, то случайный граф $G(n, p)$ подчиняется закону нуля или единицы. В данной работе мы ограничиваемся случаем $0<\alpha<1$, который представляет наибольший интерес (подробное описание результатов для случайного графа $G(n, p)$ при $p=O(1 / n)$ можно найти в [1]). Если $\alpha \in \mathbb{Q} \cap(0,1)$, то случайный граф $G\left(n, n^{-\alpha}\right)$ закону нуля или единицы не подчиняется (см. [2]).

Если случайный граф $G\left(n, n^{-\alpha}\right)$ не подчиняется $k$-закону нуля или единицы для некоторого $\alpha \in(0,1)$ и некоторого $k \in \mathbb{N}$, то говорят, что $\alpha$ является элементом спектра числа $k$. Напомним, что в [11] были рассмотрены два понятия спектра формулы первого порядка $L \in \mathcal{L}$. Первое определение возникает при рассмотрении вероятностей проведения ребра вида $p=n^{-\alpha}$. Множество $S^{1}(L)$ состоит из всех таких $\alpha \in(0,1)$, что не выполнено следующее свойство: $\lim _{n \rightarrow \infty} \mathrm{P}\left(G\left(n, n^{-\alpha}\right) \mid=L\right)$ существует и равен либо 0 , либо 1 . Второе определение возникает при рассмотрении более широкого класса вероятностей $p=n^{-\alpha+o(1)}$. Множество $S^{2}(L)$ состоит из всех таких $\alpha \in(0,1)$, что не выполнено следующее свойство: существуют $\delta \in\{0,1\}$ и $\varepsilon>0$, для которых $\lim _{n \rightarrow \infty} \mathrm{P}(G(n, p(n)) \mid=L)=\delta$ для любого $p(n) \in\left(n^{-\alpha-\varepsilon}, n^{-\alpha+\varepsilon}\right)$. Пусть $k \geqslant 1$. Обозначим $S_{k}^{1}$ и $S_{k}^{2}$ объединения множеств $S^{1}(L)$ и $S^{2}(L)$ соответственно по всем $L \in \mathcal{L}_{k}$.

В [12] доказано, что при достаточно больших $k$ множества $S_{k}^{1}$ и $S_{k}^{2}$ бесконечны. Известно также [13], что у любой предельной точки множества $S_{k}^{2}$ (множества $S_{k}^{1}$ ) существует левая полуокрестность, не содержащая точек множества $S_{k}^{2}$ (множества $S_{k}^{1}$ ). В [11] доказано, что минимальные $k_{1}$ и $k_{2}$, при которых множества $S_{k_{1}}^{1}$ и $S_{k_{2}}^{2}$ бесконечны, принадлежат множествам $\{4, \ldots, 12\}$ и $\{4, \ldots, 10\}$ соответственно. Более того, в той же работе мы оценили наименьшие и наибольшие предельные точки множеств $S_{k}^{1}, S_{k}^{2}$. Обозначим $\left(S_{k}^{1}\right)^{\prime}$ и $\left(S_{k}^{2}\right)^{\prime}$ множества предельных точек множеств $S_{k}^{1}$ и $S_{k}^{2}$

Работа выполнена при поддержке РФФИ (гранты № 13-01-00612, 15-01-03530) и гранта Президента РФ МК-2184.2014.1.

DOI: $10.4213 / \mathrm{rm} 9664$ 
соответственно. Тогда

$$
\begin{array}{rlrl}
\min \left(S_{k}^{1}\right)^{\prime} \in\left[\frac{1}{k-2}, \frac{1}{k-11}\right] & \text { при } k \geqslant 15, \\
\min \left(S_{k}^{2}\right)^{\prime} \in\left[\frac{1}{k-1}, \frac{1}{k-7}\right] & \text { при } k \geqslant 10, & \\
\max \left(S_{k}^{j}\right)^{\prime} \in\left[1-\frac{1}{2^{k-13}}, 1-\frac{1}{2^{k-1}}\right] & \text { при } k \geqslant 16, \quad j \in\{1,2\} .
\end{array}
$$

В настоящей работе мы получили новую оценку на наименьшую предельную точку спектра при малых $k$.

Теорема 1. Для любого $k \geqslant 5$ имеет место включение $1 /[k / 2] \in\left(S_{k}^{1}\right)^{\prime}$.

Тем самым мы улучшили верхнюю оценку на наименьшую предельную точку спектра $S_{k}^{1}$ при всех $k \leqslant 20$ и верхнюю оценку на наименьшую предельную точку спектра $S_{k}^{2}$ при всех $k \leqslant 12$.

Из теоремы 1 следует, что наименьшее $k$, при котором спектр $S_{k}^{1}\left(S_{k}^{2}\right)$ бесконечен, равно либо 4 , либо 5.

Кроме того, мы улучшили нижнюю оценку на наибольшую предельную точку спектра (в том числе и для малых $k$ ).

Теорема 2. Для любого $k \geqslant 8$ имеет место включение $1-1 / 2^{k-5} \in\left(S_{k}^{1}\right)^{\prime}$.

Для доказательства теорем мы для каждого $k$ (не меньшего 5 в случае теоремы 1 и не меньшего 8 в случае теоремы 2) и $m \in \mathbb{N}$ построили пример такого свойства $L$, выражаемого формулой первого порядка кванторной глубины $k$, что существует последовательность случайных графов $G\left(n_{i}, n_{i}^{-\alpha_{m}}\right)$, обладающая свойством $L$ с асимптотической вероятностью, отличной от 0 и от 1 . Последовательность $\left\{\alpha_{m}\right\}_{m \in \mathbb{N}}$ определяется следующим образом: $\alpha_{m}=1 /[k / 2]+1 /([k / 2](m+[k / 2]-1))$ в случае теоремы 1 и $\alpha_{m}=1-1 / 2^{k-5}+1 /\left(2^{k-5} m\right)$ в случае теоремы 2 . Так как в случае теоремы $1 \alpha_{m} \rightarrow 1 /[k / 2]$, а в случае теоремы $2 \alpha_{m} \rightarrow 1-1 / 2^{k-5}$ при $m \rightarrow \infty$, то из существования таких свойств следуют утверждения теорем.

\section{Список литературы}

[1] S. Shelah, J. Spencer, J. Amer. Math. Soc., 1:1 (1988), 97-115. [2] M. Е. Жуковский, А. М. Райгородский, УМH, 70:1(421) (2015), 35-88; англ. пер.: М.Е. Zhukovskii, A. M. Raigorodskii, Russian Math. Surveys, 70:1 (2015), 33-81. [3] М. Е. Жуковский, Докл. PAH, 436:1 (2011), 14-18; англ. пер.: M.E. Zhukovskii, Dokl. Math., 83:1 (2011), 8-11. [4] M. Zhukovskii, Discrete Math., 312:10 (2012), 1670-1688. [5] М. Е. Жуковский, Докл. PAH, 454:1 (2014), 23-26; англ. пер.: M.E. Zhukovskii, Dokl. Math., 89:1 (2014), 16-19. [6] М.Е. Жуковский, Матем. сб., 206:4 (2015), 13-34; англ. пер.: M.E. Zhukovskii, Sb. Math., 206:4 (2015), 489-509. [7] М. Е. Жуковский, Матем. заметки, 97:2 (2015), 203-216; англ. пер.: М. E. Zhukovskii, Math. Notes, 97:2 (2015), 190-200. [8] B. Bollobás, Random graphs, 2nd ed., Cambridge Stud. Adv. Math., 73, Cambridge Univ. Press, 2001, xviii+498 pp. [9] S. Janson, T. Łuczak, A. Ruciński, Random graphs, Wiley-Intersci. Ser. Discrete Math. Optim., Wiley-Interscience, New York, 2000, xii+333 pp. [10] Н. К. Верещагин, А. Шень, Языки и исчисления, МЦНМО, М., 2000, 286 с. [11] J. H. Spencer, M. E. Zhukovskii, "Spectra for random graphs of fixed quantifier depth", Discrete Math. (submitted). [12] J. Spencer, Combinatorica, 10:1 (1990), 95-102. [13] J. H. Spencer, Discrete Appl. Math., 30:2-3 (1991), 235-252.

\section{Максим Евгеньевич Жуковский}

(Maksim E. Zhukovskii)

Московский физико-технический институт

(государственный университет)

E-mail: zhukmax@gmail.com
Представлено В. М. Бухштабером Принято редколлегией 15.04.2015 